\title{
COVID-19 outbreak among temporary foreign workers in British Columbia, March to May 2020
}

\author{
Silvina Mema ${ }^{1 *}$, Gillian Frosst ${ }^{1}$, Kristen Hanson ${ }^{1}$, Cheryl Yates ${ }^{1}$, Amanda Anderson ${ }^{1}$, \\ Jennifer Jacobsen ${ }^{1}$, Caroline Guinard ${ }^{2}$, America Lima ${ }^{3}$, Tannis Andersen ${ }^{1}$, Melissa Roe ${ }^{1}$
}

\begin{abstract}
Background: During the coronavirus disease 2019 (COVID-19) pandemic, temporary foreign workers (TFWs) provided a critical role to maintaining the food supply in Canada, yet workers faced a number of challenges that made them particularly vulnerable to COVID-19. The objective of this study was to describe the epidemiological investigation and public health response to a COVID-19 outbreak among TFWs in an agricultural setting in British Columbia from March to May 2020.

Methods: An outbreak was declared on March 28, 2020 following detection of two cases of COVID-19 among a group of 63 TFWs employed by a nursery and garden centre. Outbreak control measures included immediate isolation of cases, case finding via outreach screening and testing, cohorting of asymptomatic workers and enhanced cleaning and disinfection. The outbreak was declared over on May 10, 2020.
\end{abstract}

Results: A total of 26 COVID-19 cases were identified among the group of TFWs; no cases were identified among local workers. Cases were primarily male (77\%) with a median age of 41 years. Symptom onsets ranged from March 8 to April 9, 2020. One case required overnight hospitalization for pneumonia.

Conclusion: This was the first COVID-19 community outbreak identified in British Columbia and the first COVID-19 outbreak identified among TFWs in Canada. This outbreak began prior to implementation of provincial and federal quarantine orders for international travellers. A provincial policy was later developed that requires TFWs to quarantine in government-funded accommodation prior to deployment to agricultural settings.
This work is licensed under a Creative Commons Attribution 4.0 International License.

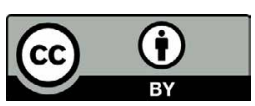

Affiliations

${ }^{1}$ Interior Health, Kelowna, BC

${ }^{2}$ Faculty of Medicine, University of British Columbia,

Vancouver, BC

${ }^{3}$ Family Physician at Turtle Lake Medical Clinic, Lake Country, BC

${ }^{4}$ British Columbia Centre for Disease Control Public Health Laboratory, Vancouver, BC

\section{*Correspondence:}

silvina.mema@interiorhealth.ca

Suggested citation: Mema SC, Frosst G, Hanson K, Yates C, Anderson A, Jacobsen J, Guinard C, Lima AU, Andersen T, Roe M. COVID-19 outbreak among temporary foreign workers in British Columbia, March to May 2020. Can Commun Dis Rep 2021;47(1):5-10. https://doi.org/10.14745/ccdr.v47i01a02

Keywords: COVID-19, outbreaks, temporary foreign workers, migrant workers, agricultural workers

\section{Introduction}

In December 2019, the novel severe acute respiratory syndrome coronavirus 2 (SARS-CoV-2) emerged in Wuhan, China and subsequently spread globally. Coronavirus disease 2019 (COVID-19) typically manifests as influenza-like illness and the virus is primarily transmitted between people through respiratory droplets and contact routes causing widespread outbreaks. In response to the rapid spread and need for a coordinated international response, the World Health Organization (WHO) declared the COVID-19 outbreak a Public Health Emergency of International Concern on January 30, 2020 and a pandemic on March 11, 2020 (1).
International travellers are at higher risk of developing and contributing to the spatial spread of COVID-19 (2). As a result, governments around the world implemented strict international travel restrictions to limit the global impacts of the virus. In Canada, certain categories of people who provided essential services were exempt from the travel restrictions, including temporary foreign workers (TFWs) who play a critical role during the crop season by contributing to the economy and maintaining the food supply $(3,4)$. TFWs are migrant workers who travel to Canada every year, typically from Latin America, to work in the agricultural sector. The Okanagan Valley in British Columbia (BC), Canada is a major centre for agriculture production and the operation of the agricultural industry in this region relies on the employment of TFWs. 
Early in the pandemic, from March to May 2020, an outbreak of COVID-19 was declared among TFWs in an agricultural setting in the Okanagan region. This outbreak provided an insight into challenges that made TFWs particularly vulnerable during the COVID-19 pandemic. These challenges may include language and cultural barriers, fear of loss of work or even deportation if disclosing symptoms, as well as variability in adequacy of living conditions including access to laundry and food, availability of wages while isolating or sick, access to medical services and coverage of medical costs and access to transportation (5).

This report describes the epidemiological investigation and public health control measures implemented to respond to the outbreak.

\section{Methods}

\section{Outbreak investigation team}

This outbreak investigation was led by the Interior Health Medical Health Officer with a team that included an epidemiologist, two communicable disease specialists with experience in contact tracing, primary care nursing staff, a nurse practitioner, two environmental health officers and a health administrator. Additional support was provided by a seasonal worker program coordinator employed by the agricultural business affected by the outbreak, a migrant support outreach worker from a local community resource centre and two local primary care physicians.

\section{Outbreak detection and case finding}

From March 24 to 27, 2020, two confirmed cases of COVID-19 were reported among a cohort of 63 TFWs employed by a nursery and garden centre in the Okanagan region. An outbreak was declared on March 28, 2020, and declared over on May 10, 2020, following a period of 28 days (two incubation periods) since the testing date of the last identified case.

For this investigation, confirmed outbreak cases were defined as nursery workers with the following:

- Laboratory-confirmed COVID-19 (6)

- Symptom onset date on or after March 1, 2020

Epidemiologically-linked (epi-linked) cases were defined as nursery workers with the following:

- $\quad$ Symptoms compatible with COVID-19

- Symptom onset on or after March 1, 2020

- A known epidemiological link to a confirmed case

Case finding activities included daily symptom checks of all TFWs and a detailed symptom screening questionnaire (see Supplemental material information) that was administered four times throughout the investigation. The surveys were administered in person via outreach medical teams. Additional data sources included medical records of cases and interviews initiated through public health follow-up of cases and contacts.
TFWs were Spanish-speaking; therefore, information and services were provided in Spanish whenever possible. Translation was provided through the Provincial Language Service (7) as well as the seasonal worker program coordinator, migrant support outreach worker and a primary care physician, all of whom were fluent in both English and Spanish.

All workers who reported symptoms compatible with COVID-19 were referred for testing. A small number of asymptomatic workers who were considered to be at highest risk of COVID-19 infection were also tested. Respiratory specimens collected as nasopharyngeal swabs underwent nucleic acid amplification testing at the regional microbiology laboratory. Extraction was performed on the STARlet liquid handling system (Hamilton) using STARmag 96 universal cartridge kits. Polymerase chain reaction (PCR) testing was carried out using the CFX96 (BioRad), using the Allplex 2019-nCoV assay targeting the E, RdRP and $\mathrm{N}$ genes following the manufacturer's instructions (Seegene).

\section{Analysis}

Reportable information about cases was available through Panorama, Interior Health's public health information system (8). An additional line list of all 63 TFWs was maintained that included detailed information on age, gender, arrival date to Canada, local accommodation location(s), specific work role(s) at the nursery and COVID-19 testing date(s) and results. Descriptive analyses were performed using Microsoft Excel 2010 and SAS version 9.4 software.

\section{Public health measures}

On the day the outbreak was declared, a site inspection was conducted by two environmental health officers to assess the work environment and living conditions of the workers. A public health order under the BC Public Health Act (9) was issued to the business operator requiring the following: enhanced cleaning of facilities accessed by employees; screening of staff, contractors and visitors; mandatory reporting of new respiratory illness among employees or contractors; and quarantine of all TFWs. This order was rescinded when the outbreak was declared over.

Case management included daily monitoring as per BC's interim guidelines for public health management of cases and contacts associated with COVID-19 (10). Daily reporting by the seasonal worker program coordinator to Interior Health's Communicable Disease Unit was requested and included the health status of cases and contacts. Any newly symptomatic individuals were reported to the Communicable Disease Unit for public health follow-up. A protocol was established to safely transport any individuals requiring healthcare services to and from healthcare facilities when use of an ambulance was not indicated. All workers also had access to virtual appointments with a primary care physician, if needed.

TFWs lived in employer-provided, shared accommodation consisting of five houses and 11 trailers across five geographically distinct sites. Symptomatic individuals were immediately isolated, 
initially within existing housing locations. Due to the increasing number of cases and limited availability of single person self-contained space, symptomatic workers were later relocated to individual hotel rooms. During the outbreak, none of the TFWs were permitted to go into the community or interact with other workers outside of their geographically distinct site.

Within shared housing locations, a rotation schedule was applied for use of common areas to ensure physical distancing. Enhanced cleaning and disinfection of common areas within each housing site, such as kitchens and bathrooms, was implemented. Food and other essential supplies were delivered to each housing unit throughout the outbreak period. Communication with the workers occurred primarily by phone and email, and a mobile app (WhatsApp Inc.) was used to provide messaging to the workers and to conduct the daily symptom checks. Full personal protective equipment was worn by healthcare providers and managerial staff during site visits. Workers had access to phones to maintain connections with family and friends and received regular pay during the quarantine period.

To enable the business to maintain operations, asymptomatic workers were divided into cohorts, both in shared accommodation and at worksites, and enhanced control measures, such as portable hand washing stations and tools for self-use by individual workers, were introduced. In addition, physical distancing and staggering of breaks to prevent congregation were recommended. Lastly, a 72-hour quarantine or spray clean with 10:1 water-bleach solution was implemented to prevent transmission of SARS-CoV-2 from handled products, such as potted trees/plants, prior to being shipped to the retail store.

A medical outreach team conducted four site visits during the investigation. The team included a combination of a nurse practitioner, a registered nurse and/or a physician. The purpose of the visits was case finding, testing, monitoring of cases and education of workers and other employees. During each visit, the medical team asked all TFWs to complete the symptom screening questionnaire. The team collected respiratory specimens and conducted physical assessments of symptomatic workers including those experiencing only mild symptoms (excluding previously identified cases). In the initial stage of the investigation, an additional 12 local workers who interacted with the foreign workers were also screened to determine the extent of the outbreak among the nursery's employees. These workers were also asked to self-isolate for 14 days. During their site visits, the outreach team visited the isolated cases as part of their monitoring and to ensure their well-being. Furthermore, the visits allowed for an opportunity to educate and emphasize prevention measures with the workers and managerial personnel.

\section{Results}

\section{Epidemiologic investigation}

On March 23, 2020, the Interior Health Medical Health Officer was notified by the Ministry of Health and the Ministry of Agriculture of suspected COVID-19 in a hospitalized TFW. Laboratory-confirmation was received the following day. Routine contact tracing by public health identified one household contact who was also symptomatic and was initially considered an epilinked case (later confirmed). On March 27, 2020, a second worker from the same nursery/garden centre presented to the hospital and was confirmed to have COVID-19. This individual resided in a separate household with no reported contact with the first confirmed case. This suggested more widespread transmission of COVID-19 among the worker population and prompted the Medical Health Officer to declare the outbreak. Seventeen additional confirmed cases were identified following the first health outreach team visit on March 30, 2020.

A total of 26 COVID-19 cases were identified, including 23 confirmed and three epi-linked cases. All cases were reported among TFWs. Thirty-one of the 63 foreign workers were tested over the course of the investigation resulting in $74 \%$ positivity (confirmed cases only) among those tested. The epi-linked cases tested negative for the SARS-CoV-2 virus; however, these individuals were managed as cases, given exposure and symptom histories compatible with COVID-19. No cases were identified among the 12 local workers (67\% males; mean age 43 years; median age 44.5 years) possibly due to control measures implemented by the business owner early on, which limited interaction between the local workers and TFWs.

Characteristics of cases are shown in Table 1. The majority of outbreak cases were male (77\%) with a mean and median age of 41 years. The age and sex distribution of cases reflected that of the full cohort of 63 TFWs. Symptom onsets ranged from March 8 to April 9, 2020. Symptom onsets were not available for five (19\%) cases. One case required overnight hospitalization for pneumonia and all cases recovered fully from their illness. Figure 1 shows an epidemic curve of outbreak cases by episode date from March 8 to May 11, 2020. Key investigation dates are also shown.

\section{Implementation of worker cohorting}

All 63 TFWs were considered to be potentially exposed to COVID-19 and were therefore required to isolate. Asymptomatic workers were separated into cohorts within shared accommodations as isolation of all workers in single rooms with private bathrooms (i.e. in hotel rooms) was neither feasible nor practical. Employer-provided accommodations were clean, well maintained and stocked with essential items, such as liquid soap, paper towel and cleaning supplies. Initially, all asymptomatic workers were required to self-isolate in their rooms within the shared housing. Later on, when the outbreak control measures had been fully implemented, asymptomatic workers returned to work within their geographical sites with physical distancing, 
Table 1: Characteristics of COVID-19 cases included in the outbreak investigation $(\mathrm{N}=26)$

\begin{tabular}{|c|c|c|}
\hline $\begin{array}{l}\text { Characteristics of } \\
\text { COVID-19 cases }\end{array}$ & $\begin{array}{c}\text { Number of } \\
\text { cases }\end{array}$ & $\%$ of total \\
\hline \multicolumn{3}{|l|}{ Cases } \\
\hline Confirmed & 23 & $88 \%$ \\
\hline Confirmed epi-linked & 3 & $12 \%$ \\
\hline Total & 26 & $100 \%$ \\
\hline \multicolumn{3}{|l|}{ Symptoms } \\
\hline Onset date range & $\begin{array}{l}\text { March 8-April 9, } \\
2020\end{array}$ & $\begin{array}{l}\text { March 8-April 9, } \\
2020\end{array}$ \\
\hline Asymptomatic/not provided & 5 & $19 \%$ \\
\hline \multicolumn{3}{|l|}{ Demographics } \\
\hline Males & 20 & $77 \%$ \\
\hline Females & 6 & $23 \%$ \\
\hline \multicolumn{3}{|l|}{ Age group (years) } \\
\hline Younger than 35 & 5 & $19 \%$ \\
\hline $35-39$ & 5 & $19 \%$ \\
\hline $40-44$ & 8 & $31 \%$ \\
\hline 45 and older & 8 & $31 \%$ \\
\hline Hospitalized & 1 & $4 \%$ \\
\hline
\end{tabular}

Figure 1: Epidemic curve of outbreak COVID-19 cases by episode date ${ }^{a}$, March 8 to May 11, 2020 (N=26)

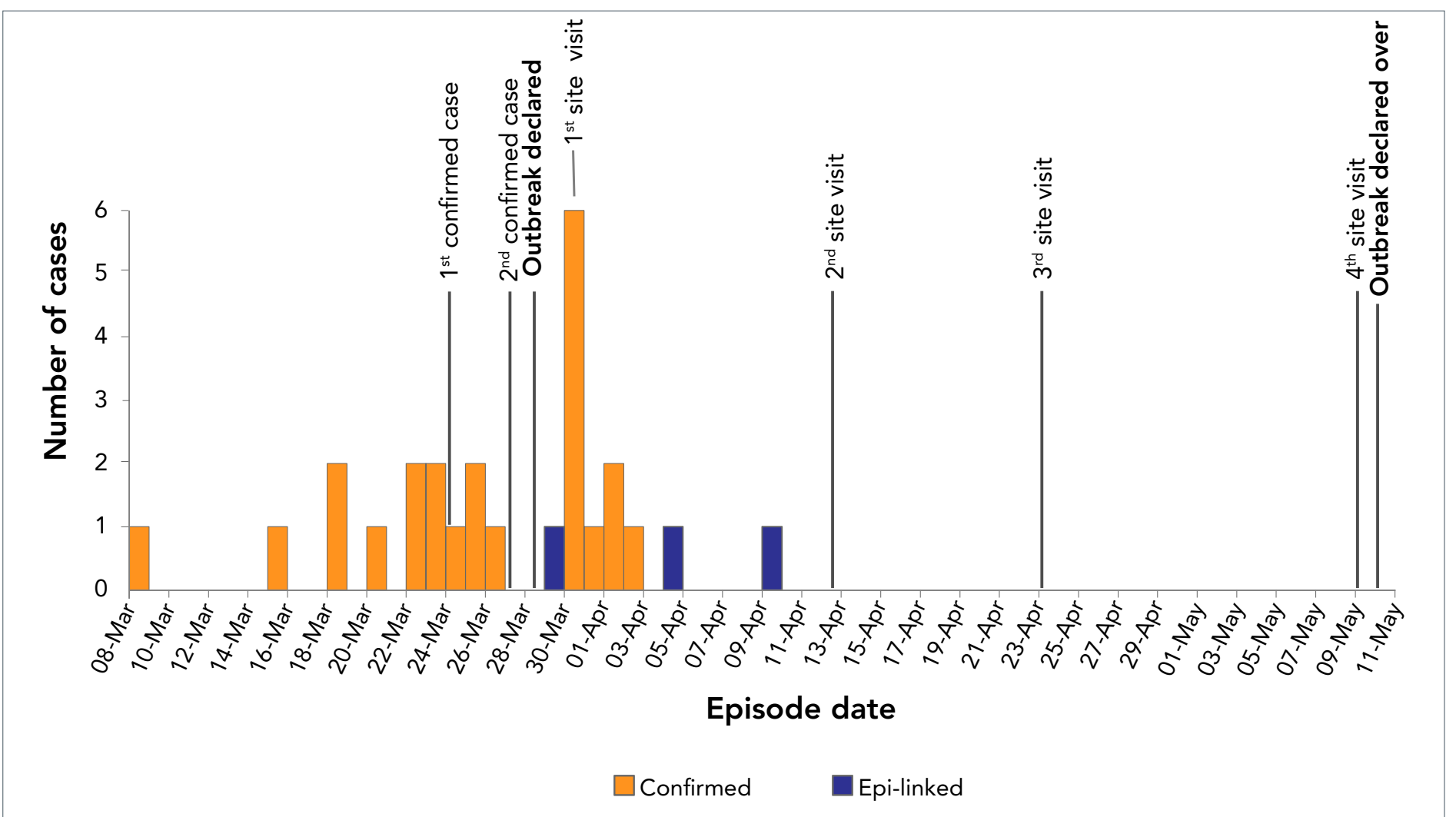

Abbreviation: COVID-19, coronavirus disease 2019

a Episode date based on symptom onset $(n=21)$; if not available, then specimen collection date ( $n=5$, all March 30 ) while continuing to isolate in their households when not at work. Their roles consisted of activities in fields and greenhouses that were geographically separated from other nursery facilities and did not include the retail space open to the public. Of note, of the five geographically distinct accommodation sites, only one site did not have any COVID-19 cases identified among the workers (five TFWs). This accommodation/work site was considered more geographically isolated than the other locations with limited interaction with the other workers prior to the outbreak detection.

\section{Discussion}

This report summarizes a COVID-19 outbreak affecting a group of TFWs in an agricultural setting. The outbreak was declared shortly after WHO assessed COVID-19 as a pandemic and in the early days of Canada's epidemic. This was the first COVID-19 community outbreak identified in BC and the first COVID-19 outbreak identified among TFWs in Canada. Since this time, other outbreaks affecting agricultural workers have been reported (11-13).

TFWs are generally a healthy workforce. Thus, two COVID-19 cases from different worker households requiring hospital attention suggested the "tip of the iceberg" in terms of the potential for other milder cases in the worker group. This 
prompted the outbreak declaration. Subsequent screening of all workers with a symptom screening questionnaire resulted in the detection of several additional cases who, given that their symptoms were mild, had not sought care.

During the investigation, we identified a number of barriers to care among TFWs. Despite daily symptom checks, delayed reporting of symptoms was noted for some individuals and may have been due to health literacy and language barriers, but also fear about missed work, lost wages and lack of healthcare coverage. Access to multilingual service providers was an important factor in delivering culturally safe and appropriate care. Spanish-speaking service providers were included in each outreach visit, which minimized the need for employerprovided staff to translate and potentially deter the workers from fully sharing health concerns. TFWs also had access to a local physician from the same country of origin as the workers, and access to telehealth services with this physician was facilitated by billing changes (14) triggered by the COVID-19 pandemic.

TFWs affected by this outbreak arrived to Canada between January 16 and March 12, 2020, just prior to the provincial and federal quarantine orders for international travellers. On March 17, BC's Provincial Health Officer introduced an order for international travellers returning to, or arriving in, $B C$ to self-isolate for 14 days (15). A week later, on March 24, the federal government enacted a similar mandatory quarantine order for returning travellers (16). At the time of writing this report (July 2020), all TFWs entering BC are required to self-isolate in government-managed accommodation for 14 days prior to their deployment to farms. During this time, employers are responsible for paying workers for a minimum of 30 hours per week and provincial funding is available for hotel and other supporting costs. Workers are also screened before departure from their country of origin and upon arrival in Canada (17). In addition, national guidelines have been developed to assist employers of TFWs in understanding their role in helping to protect the health and safety of their employees in the context of COVID-19 (18).

\section{Strengths and limitations}

Strengths of this outbreak investigation include the collaboration between the affected business and health officials throughout the course of the investigation, the coordinated response involving both internal and external stakeholders, and the occurrence of this outbreak in a well-defined cohort of workers. The nursery management was proactive in terms of having measures in place for prevention and early identification of cases in the time leading up to the outbreak declaration. The employer also provided wages and essential supplies to workers throughout the period of isolation as well as singlesite accommodation as required for symptomatic workers. A limitation of this investigation was that we did not test all of the 63 TFWs for SARS-CoV-2. We tested only those who were symptomatic and a small number of other workers that we felt were at highest risk of infection given their potential exposure to a confirmed case. At the time of the outbreak, the incidence of COVID-19 was rapidly increasing in $B C$ and there were concerns about a potential shortage of nasopharyngeal swabs. It is likely that broader testing within the foreign worker population would have identified further cases. Risk factor analysis was also limited in this investigation. When the outbreak was detected, significant transmission had already occurred within the TFW cohort. Accommodation and work site groups were also rearranged during the investigation to minimize exposures and transmission. As a result, we were unable to identify particular locations that were risk factors for symptomatic infection and were limited in our ability to identify how the infection might have originated and spread through the group. There is potential for whole genome sequencing to provide additional insights into the disease transmission pattern in a future phase of the outbreak analysis.

\section{Conclusion}

TFWs have had unique risks during the COVID-19 pandemic as demonstrated by this outbreak, which occurred in an agricultural setting in BC. Transmission of COVID-19 was confirmed through prompt identification and declaration of an outbreak and repeated symptom screening followed by targeted testing. Immediate quarantine of affected workers, comprehensive follow-up of cases and contacts, and mobilization of an outreach team were effective strategies to manage and control this outbreak. These measures were implemented while still allowing for some continued business operations. Provincial and federal orders and guidance have since been developed to reduce outbreak risk in agricultural settings and to protect the health and safety of both workers and Canadians in the context of the pandemic.

\section{Authors' statement}

SCM - Conceptualization

SCM, GF, KH, CY, AA, JJ, AUL, TA, MR - Investigation

SCM, GF - Writing original draft

SCM, GF, KH, CY, AA, JJ, CG, AUL, TA, MR - Writing review and editing

\section{Competing interests}

None.

\section{Acknowledgements}

We would like to acknowledge all individuals at Interior Health who supported this outbreak investigation. We would also like to thank J Robles from KCR Community Resources for supporting the provision of culturally safe outreach care. 


\section{Funding}

This work was supported by Interior Health.

\section{Supplemental material}

Symptom Assessment Survey (English) (https://www.canada. ca/content/dam/phac-aspc/documents/services/reportspublications/canada-communicable-disease-report-ccdr/monthlyissue/2021-47/issue-1-january-2021/ccdrv47i01a02as-eng.pdf)

Encuesta de Evaluación de Salud (Español) (https://www. canada.ca/content/dam/phac-aspc/documents/services/reportspublications/canada-communicable-disease-report-ccdr/monthlyissue/2021-47/issue-1-january-2021/ccdrv47i01a02bs-spa.pdf)

The content and view expressed in this article are those of the authors and do not necessarily reflect those of the Government of Canada.

\section{References}

1. World Health Organization. Coronavirus disease (COVID-19) pandemic. Geneva (Switzerland): WHO; 2020 (accessed 2020-06-22). https://www.who.int/emergencies/diseases/ novel-coronavirus-2019

2. Government of Canada. Coronavirus disease (COVID-19): Travel restrictions, exemptions and advice. Government of Canada (updated 2020-10-15; accessed 202007-05). https://www.canada.ca/en/public-health/ services/diseases/2019-novel-coronavirus-infection/ latest-travel-health-advice.html

3. Government of British Columbia. List of COVID-19 Essential Services. Government of BC (updated 2020-06-12; accessed 2020-06-22). https://www2.gov.bc.ca/gov/content/safety/ emergency-preparedness-response-recovery/covid19-provincial-support/essential-services-covid-19

4. Agriculture and Agri-Food Canada. Keeping Canadians and workers in the food supply chain safe. AAFC (updated 2020-04-13; accessed 2020-06-23). https://www.canada.ca/ en/agriculture-agri-food/news/2020/04/keeping-canadian s-and-workers-in-the-food-supply-chain-safe.html

5. Government of Canada. Vulnerable populations and COVID-19. Government of Canada (updated 2020-1001; accessed 2020-06-22). https://www.canada.ca/en/ public-health/services/publications/diseases-conditions/ vulnerable-populations-covid-19.html

6. BC Centre for Disease Control. COVID-19 (Novel Coronavirus): Case definitions. BCCDC; 2020 (accessed 2020-06-22). http://www.bccdc.ca/health-professionals/ clinical-resources/case-definitions/covid-19(novel-coronavirus)
7. British Columbia Provincial Health Services Authority. Provincial Language Service. PHSA; 2020 (accessed 202006-22). http://www.phsa.ca/our-services/programs-services/ provincial-language-service

8. BC Centre for Disease Control. COVID-19 Case Report Form. BCCDC (updated 2020-10-15; accessed 2020-06-22). http://www.bccdc.ca/Documents/COVID-19_Case_Report_ Form.pdf

9. Government of British Columbia. Public Health Act (accessed 2020-06-22). https://www2.gov.bc.ca/gov/ content/health/about-bc-s-health-care-system/legislation/ public-health-act

10. BC Centre for Disease Control. Public Health Management: Surveillance and guidance for public health management of COVID-19. BCCDC; 2020 (accessed 2020-06-22).

http://www.bccdc.ca/health-professionals/clinical-resources/ covid-19-care/public-health-management

11. Chatham-Kent Public Health. News Release; April 27, 2020 (accessed 2020-06-22). https://ckphu.com/wp-content/ uploads/2020/04/Outbreak-Greenhill-Produce.pdf

12. Windsor-Essex County Health Unit. News Release: May 26th Public Health Updates Related to Coronavirus (COVID-19) (accessed 2020-06-22). https://www.wechu.org/ newsroom/news-release-may-26th-public-health-updates-re lated-coronavirus-covid-19

13. Windsor-Essex County Health Unit. News Release: June 12th Public Health Updates Related to Coronavirus (COVID-19). WECHU (updated 2020-06-12; accessed 2020-06-22). https://www.wechu.org/newsroom/news-release-june12th-public-health-updates-related-coronavirus-covid-19

14. Doctors of BC. Billing changes - COVID-19. News release: June 5, 2020 (accessed 2020-07-05). https://www. doctorsofbc.ca/news/covid-19-temporary-billing-changes

15. Government of British Columbia. Notice to people who have been or have likely been exposed to SARS-CoV-2 (Class). Government of BC (updated 2020-03-17; accessed 2020-06-22). https://www2.gov.bc.ca/assets/ gov/health/about-bc-s-health-care-system/office-o f-the-provincial-health-officer/reports-publications/covid19-pho-class-order-travellers.pdf

16. Government of Canada. Orders in Council - Search (PC Number 2020-0175). Government of Canada (updated 2020-03-24; accessed 2020-06-22). https://orders-in-council. canada.ca/attachment.php?attach $=38989 \&$ lang $=$ en

17. Government of British Columbia. Temporary foreign farmworkers must self-isolate. Government of BC (updated 2020-10-23; accessed 2020-07-05). https://www2.gov. bc.ca/gov/content/industry/agriculture-seafood/covid-19response/temporary-foreign-farmworkers

18. Employment and Social Development Canada. Guidance for employers of temporary foreign workers regarding COVID-19. Government of Canada (updated 2020-0422; accessed 2020-07-05). https://www.canada.ca/en/ employment-social-development/services/foreign-workers/ employer-compliance/covid-guidance.html 